\title{
Modelling of Levitation Melting using a Fixed Mesh Method
}

\author{
D. Hectors, E. Toorman, K. Van Reusel
}

\begin{abstract}
Numerical modelling is indispensable in the design of levitation melting equipment. The shape and the position of the levitated molten metal are very important for the power transfer from the coil towards the molten metal. This paper verifies whether the shape and position of this molten metal can be determined using the level set method.
\end{abstract}

\section{Introduction}

Levitation melting is a technique based on the induction of eddy currents in a metallic sample by an alternating electromagnetic flux. These eddy currents heat the sample by Joule effect. The induced currents give cause to Lorentz forces at the same time. When the magnetic field is generated by a suitably shaped coil, the forces may attain a sufficient magnitude to levitate the specimen. Simultaneously, the molten metal droplet deforms, due to the "magnetic pressure". The shape and the position of the liquid metal-air interface are very important for the coupling between the inductor and the levitated load. The rotational component of the levitation force, together with the thermal gradients in the liquid metal (causing convection flows), produce a stirring effect in the droplet.

The main advantage of levitation melting is that the metal is molten without contact. The applications of this technique are based on this main advantage: measurement of thermophysical properties of liquid metals [1,2], elaboration of materials that require a high degree of purity [3], processing of strongly reactive metals or alloys, and investigation of solidification of undercooled melts [4].

Numerical modelling is indispensable in the design of levitation melting equipment. The shape and the position of the molten metal is very important for the power transfer from a coil or inductor towards the molten metal. Results from numerical models tracking the position and the shape of the interface have recently been published [5]. In this publication [5] the tracking of the interface is based on a moving mesh method (ALE).

This paper describes another technique for tracking the interface, using a fixed mesh method (level set). The first section explains the level set method generally. Section two shows some results obtained by using this method for levitation melting and compares it with the ALE method. Finally, some conclusions are given.

\section{General description of the level set method}

The level set method is a technique to represent moving interfaces or boundaries using a fixed mesh. The level set method is very similar to the volume of fluid (VOF) technique [6]. The level set method however has a higher order of accuracy in comparison to VOF [7]. The major drawback of the level set method used to be that it was not conservative, but this problem has been solved [7]. 


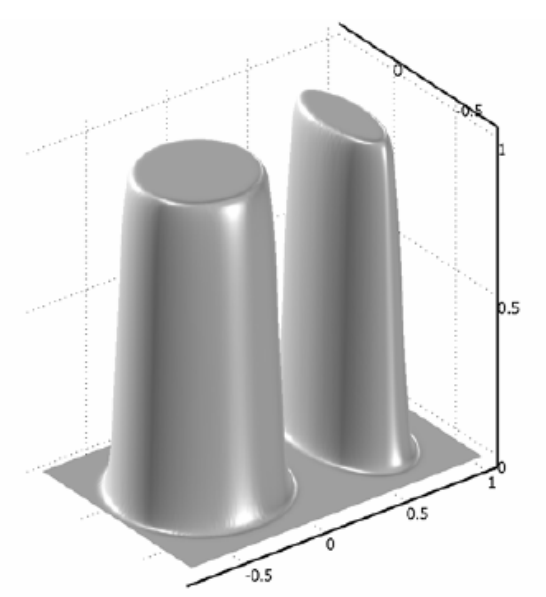

Fig.1. Surface plot of a level set function [8]

The level set method is useful for problems where the computational domain can be divided into two domains separated by an interface [8] (Fig.1). The interface is represented by a certain "level set" or "isocontour" of a globally defined function, the "level set function" $(\Phi)$. This function $\Phi$ is a smooth step function equal to zero in one domain and equal to one in the other domain. Across the interface, there is a smooth transition from zero to one. The interface is defined by the 0.5 isocontour, or the level set, of $\Phi$. By using the level set method, the following equation is solved in combination with the incompressible Navier-Stokes equations:

$$
\frac{\mathrm{d} \Phi}{\mathrm{dt}}+\mathbf{u} \cdot \nabla \Phi=\gamma \nabla \cdot\left(\varepsilon \nabla \Phi-\Phi(1-\Phi) \frac{\nabla \Phi}{|\nabla \Phi|}\right)
$$

The terms on the left hand side gives the correct motion of the interface, while the right hand side terms are necessary for numerical stability. Stabilization is necessary because (1) is a hyperbolic equation [6]. In (1), $\mathbf{u}$ is the velocity field, $\varepsilon$ is a parameter that determines the thickness of the region where $\Phi$ goes smoothly from zero to one. The parameter $\gamma$ determines the amount of reinitialization or stabilization of the level set function. A more general description of the level set method can be found in [9] and [10].

\section{Application of level set in levitation melting}

The level set method is available in the commercial finite element software Comsol [11]. This software has been used for modelling levitation melting, tracking the liquid metalair interface of a molten droplet at constant temperature, levitated by a current conducting coil. In our situation, we are interested in the deformation and movement of a liquid metal droplet, levitated by a conical coil with one winding. The 2D axisymmetric geometrical set-up of the model can be seen on Fig.2. The initial shape of the metal is a sphere. The surrounding airbox is defined bigger than shown on the figure, which is a zoom.

The electromagnetic model uses the time-harmonic axisymmetric electromagnetic diffusion equation of the scalar variable Az. This equation is solved for the whole geometry of the model. The impedance boundary condition is used on the coil, since we are not interested in the internal magnetic fields within the coil, and this way is much easier to mesh at the high frequency of $550 \mathrm{kHz}$. The impedance boundary condition is not used on the metal though. Otherwise, we would not be able to simulate the stirring of the liquid metal. 


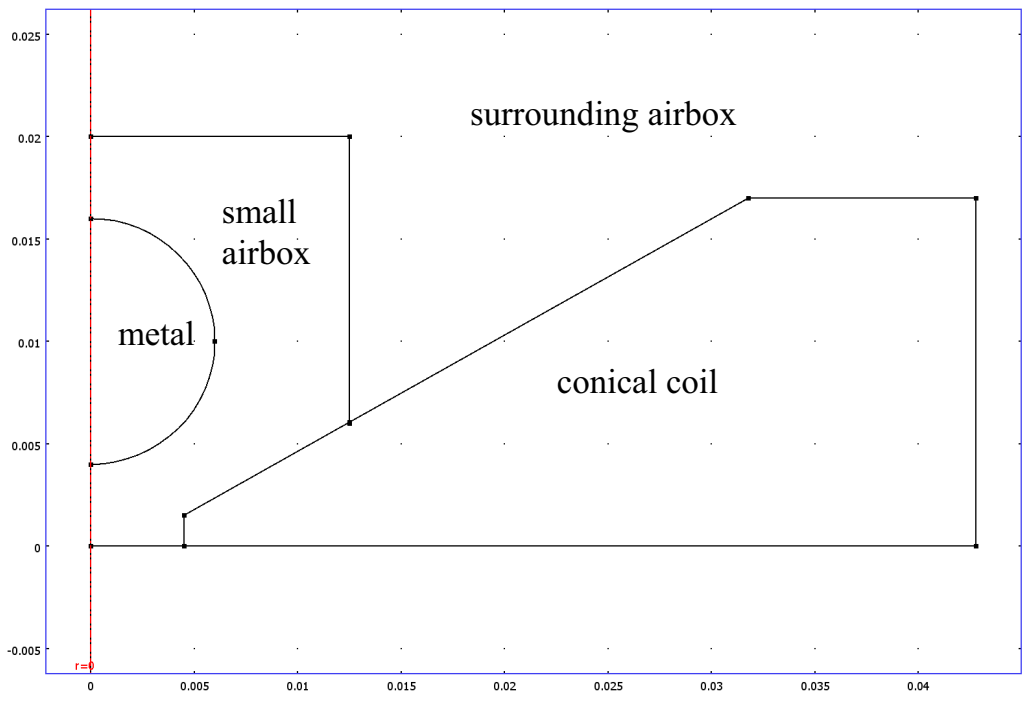

Fig.2. Geometrical set-up

The electromagnetic module is coupled with the level set module, in which there are 4 unknowns: velocity components $\mathrm{u}$ and $\mathrm{v}$, pressure $\mathrm{p}$, and level set function $\Phi$. The level set module is solved for the metal domain, and a small part of the airbox (small airbox on Fig.2). Last part is kept as small as possible, to avoid to solve the Navier-Stokes equations for too much nodes, but is made sufficiently large to surely contain the liquid deformation. The metal and the small airbox need to be meshed finely, in order to track the interface well. This fine mesh (Fig.3) allows the level set interface to be small.

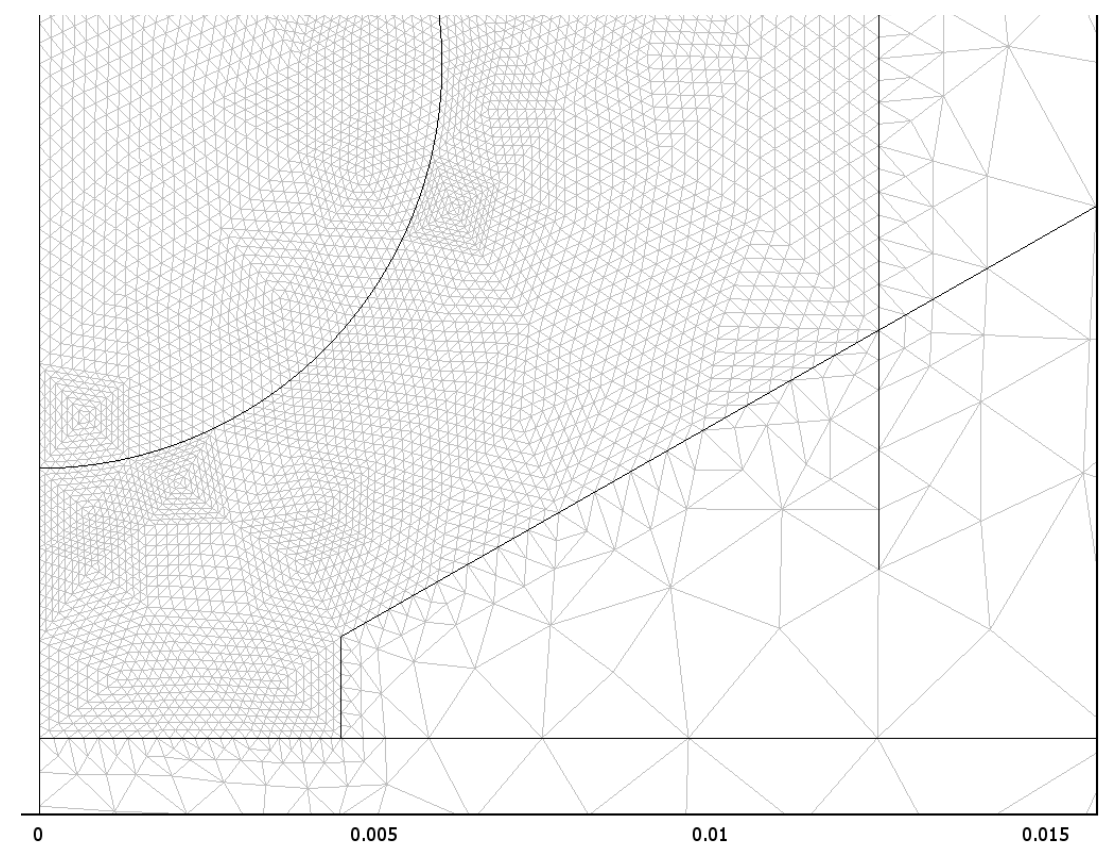

Fig.3. Mesh

The Lorentz force and gravity are entered as volume forces. The surface tension force is entered as a force on the metal-air boundary. The turbulent viscosity is taken a factor 500 larger than the laminar viscosity, like it is standard practice in laminar flow modelling. The level set equation (1) is solved transiently together with the Navier-Stokes equations. At every time step, the electromagnetic diffusion equation is solved time-harmonically. 
The result of the steady-state liquid metal-air interface shape obtained by the level set method is given in Fig.4. The shape looks very realistic.

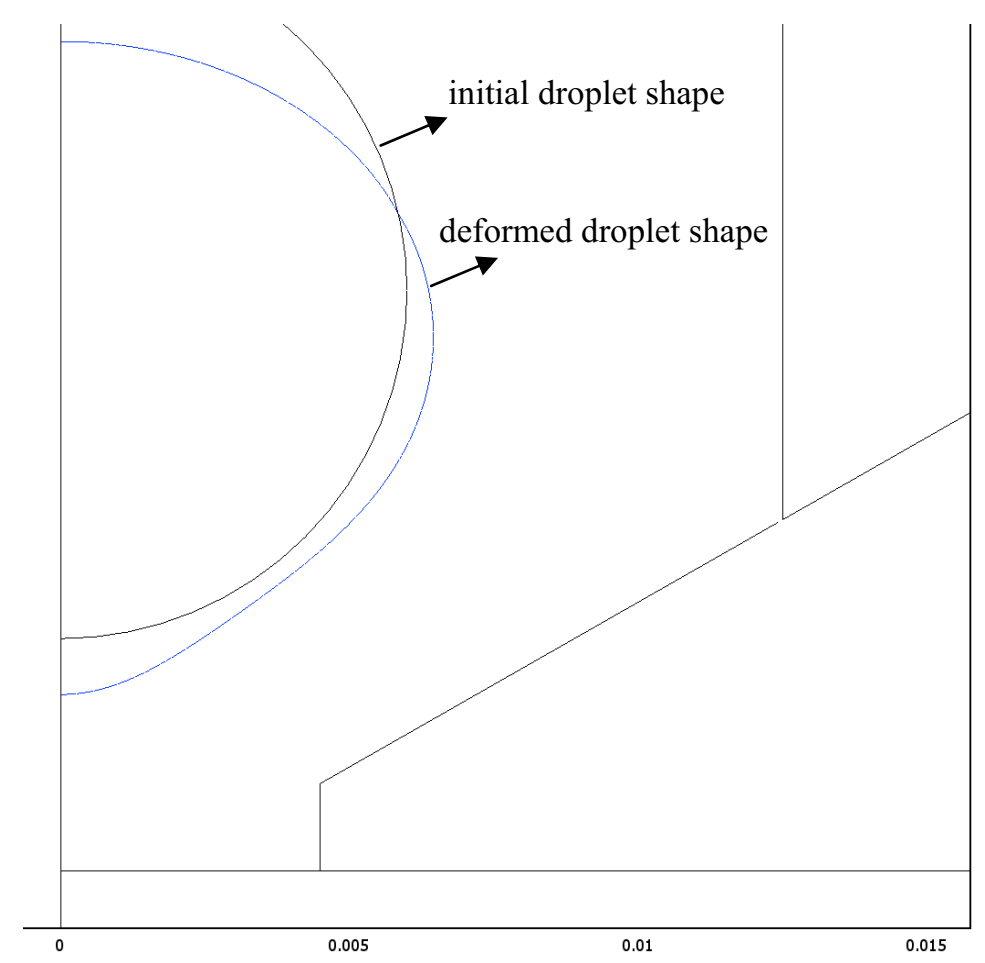

Fig.4. Regime deformed droplet shape

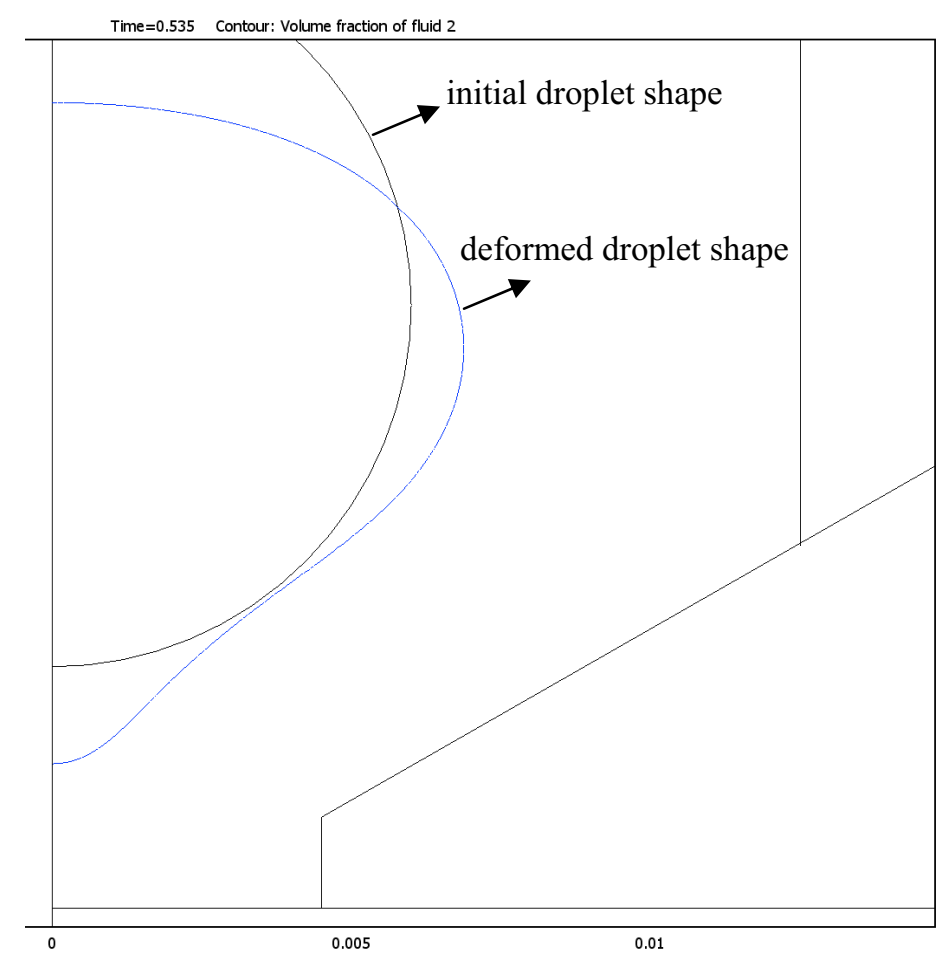

Fig.5. Regime deformed droplet shape with other surface tension

The model allows to investigate the variation of different parameters (inductor geometry, metal characteristics ...). On Fig.5, the surface tension coefficient is reduced with a factor of 4 , which can clearly be seen on the steady state shape. 
The regime droplet shape is obtained after a transient solve. Fig.6 shows the average movement of the lower part of the droplet. It can clearly be seen that the droplet has a damped oscillation movement towards a steady-state position, which is described in literature [1]. After about $1 \mathrm{~s}$, the steady-state is reached.

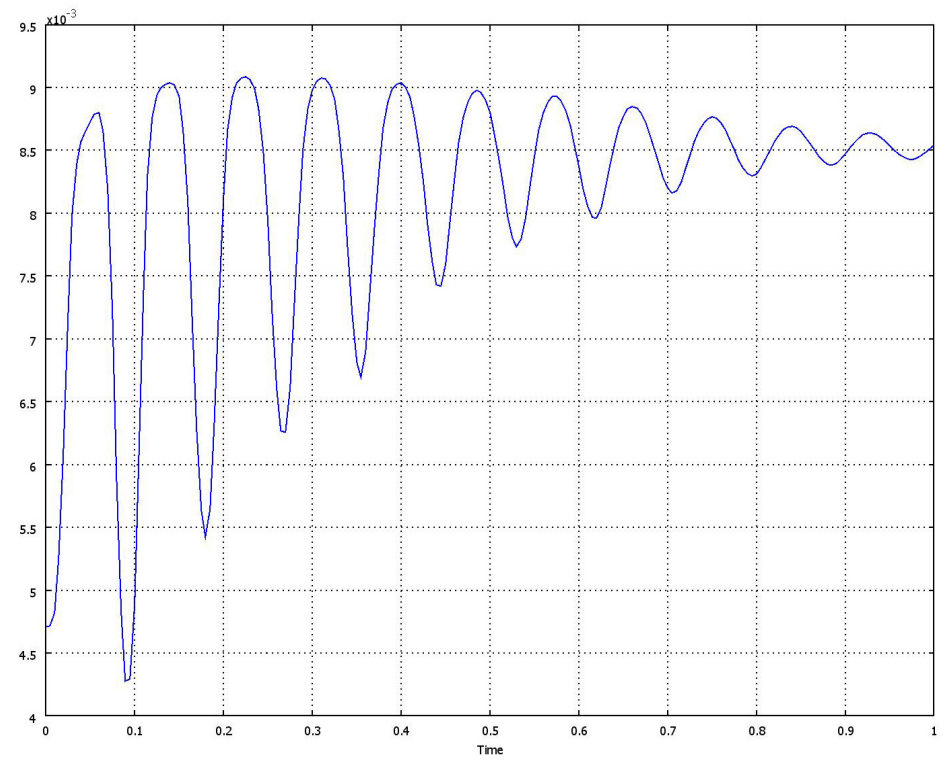

Fig.6. Transient behaviour of droplet

The stirring in the liquid metal due to Lorentz forces can be seen on Fig.7. The twophase flow can be seen on the figure.

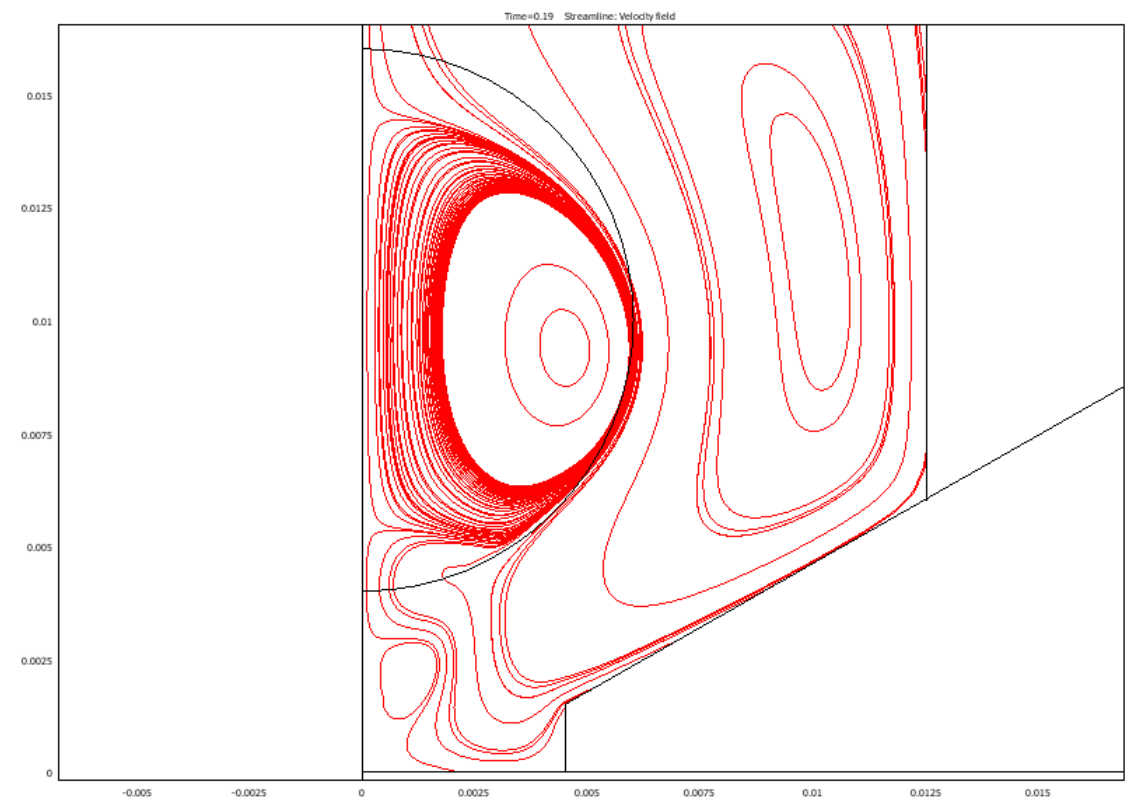

Fig.7. Streamlines of the velocity field of the two phase flow

Compared to the ALE-method, the level set method has several advantages and disadvantages:

Advantages:

- It is much easier to implement the surface tension force, and thus easier to obtain a result. 
- Fewer unknowns need to be solved for each triangle.

Disadvantages:

- The Navier-Stokes equations also need to be solved for a part of the airbox.

- The mesh needs to be finer.

- The viscosity and density vary smoothly from one fluid to the other. This is not the case in reality.

\section{Conclusions and future work}

It is possible to track the liquid metal - air interface in levitation melting, using the level set method. The results look very realistic. The models allow to investigate the behaviour of the levitated molten droplet. A next step is to compare the achieved results with position and shape measurements.

\section{References}

[1] Egry, I.: Physical property measurement of liquid metals at high temperatures under microgravity. Materials Transactions, Vol. 45, 2004, pp. 3235-3240.

[2] Lohöfer, G.: Electrical resistivity measurement of liquid metals. Measurements Science and Technology, Vol. 16, 2005, pp. 417-425.

[3] Gillo, P.: Cold crucible induction melting: theory and applications. Int. Symposium on Heating by Internal Sources, Padua, 2001.

[4] Yasuda, H., Ohnaka, I., Fujita, S., Tamura, Y., Mizuguchi, T., Nagira, T.: Dynamics and solidification behavior of the metallic melt levitated by simultaneous imposition of alternating and static magnetic fields. Joint $15^{\text {th }}$ Riga and $6^{\text {th }}$ PAMIR Int. Conference on Fundamental and Applied MHD, Riga, 2005.

[5] Ernst, R., Garnier, C., Petitpas, P., Trassy, C.: $2 D$ and $3 D$ numerical modelling of a cold crucible for optimising of industrial processes. Int. Symposium on Heating by Electromagnetic Sources, Padua, 2007.

[6] Coppola-Owen, A., Codina, R.: A finite element model for free surface flows on fixed meshes. Int. J. Numer. Meth. Fluids, Vol. 54, 2007, pp. 1151-1171.

[7] Olsson, E., Kreiss, G.: A conservative level set method for two phase flow. J. Comp. Phys., Vol. 210, 2005, pp. 225-246.

[8] Chemical Engineering user's guide, Comsol Multiphysics.

[9] Osher, S., Fedkiw, R.: Level Set Methods and Dynamic Implicit Surfaces. Springer-Verlag, Berlin, 2003.

[10] Sussman, M., Almgren, A., Colella, J., Howell, L., Welcome, M.: An adaptive level set approach for incompressible two phase flows. Journal of Computational Physics, Vol. 148, 1999, pp. 81-124.

[11] www.comsol.com

\author{
Authors \\ Ir. Ing. Hectors, Dietrich \\ Research group Electrical Energy (ELECTA) \\ Department Electrical Engineering (ESAT) \\ K.U.Leuven \\ Kasteelpark Arenberg 10 \\ 3001 Heverlee, Belgium \\ E-mail: dietrich.hectors@esat.kuleuven.be
}

Prof. Ir. Van Reusel, Koen

Research group Electrical Energy (ELECTA)

Department Electrical Engineering (ESAT)

K.U.Leuven

Kasteelpark Arenberg 10

3001 Heverlee, Belgium

E-mail: koen.vanreusel@esat.kuleuven.be
Prof. Dr. Ir. Toorman, Erik

Hydraulics Laboratory

Department of Civil Engineering

K.U.Leuven

Kasteelpark Arenberg 40

3001 Heverlee, Belgium

E-mail: erik.toorman@bwk.kuleuven.be 